\title{
Fruitfly centre spreads its wings
}

\author{
The world's main supplier of exotic Drosophila species has had a poor \\ record for customer service. But, as Rex Dalton found, it is having a facelift \\ that should boost studies of evolutionary genetics.
}

S ix years ago, evolutionary biologist Greg Spicer of San Francisco State University was gearing up for a comparative study of developmental genetics. $\mathrm{He}$ needed stocks of 50 different species of the fruitfly Drosophila, and placed his order with the National Drosophila Species Stock Center, then based at Bowling Green State University in Ohio. 'Sorry,' replied the centre's operators - there was a strict limit of ten species per request. So Spicer had to concoct a Machiavellian subterfuge using overnight express mail services and colleagues at other universities to acquire the other 40 species.

Then, when the vials of flies began arriving, Spicer discovered another problem: as many as three species in some batches of ten had been identified incorrectly - forcing him to go through the whole charade again. "The centre was awful," complains Spicer, one of several fruitfly biologists who found that its standards of customer service left much to be desired. A leading Drosophila geneticist adds: "It is a unique resource, but it hasn't been very well curated over the past few years."

But fruitfly researchers, and officials with the US National Science Foundation (NSF), which funds the centre, hope that such problems are a thing of the past. In October, the centre's colonies started moving to the University of Arizona at Tucson. There, the centre will be operated by Therese Markow, professor of ecology and evolutionary biology and director of the university's Center for Insect Science. "We plan to expand services considerably," she promises. Limits to the number of species that scientists can order have been scrapped. And Markow's team is working to purify the colonies, to ensure that researchers receive the exact species they order.

Drosophila has long been one of the most important model organisms for studying genetics and development. Much of this work involves one species: D. melanogaster. But the genus contains close to 2,000 species, and this enormous diversity also makes it ideal for comparative and evolutionary studies. Different Drosophila species live in different climates and eat different things; some are able to exploit unusual food sources because they can detoxify noxious chemicals. Decades of genetic studies on $D$. melanogaster, plus the publication of its complete genome sequence earlier this year ${ }^{1}$, have laid a solid foundation for making comparisons with and between other members of the genus to uncover the genetic basis of particular adaptive traits. "If you know the genetics of one species then you can ask about genetic differences in the others," says Markow. "You can also experiment with these animals in ways you can't with larger animals."

As well as studies that seek to identify the genetic changes that lie behind the evolution of specific adaptations, comparisons between Drosophila species can provide a route into the more fundamental territory of evolutionary genetics. Active areas of inquiry include the evolution of sex chromosomes ${ }^{2}$ and the organization of the genome $e^{3}$. Comparative studies of Drosophila have also been instrumental in casting doubt on the widely quoted theory that sexual reproduction evolved primarily to help purge the genome of harmful mutations. "Drosophila offers some wonderful opportunities," says Brian Charlesworth, an evolutionary geneticist at the University of Edinburgh.

\section{A thankless task}

The National Drosophila Species Stock Center should be an invaluable resource for evolutionary genetic studies. It maintains colonies of some 1,400 strains of fruitfly representing 265 species. And although it is billed as a national centre, it serves researchers worldwide - there is no comparable facility outside the United States. But the centre's poor performance in recent years has frustrated many of the scientists it was established to serve.

Fly collections for the stock centre began in the 1940s at the University of Texas at

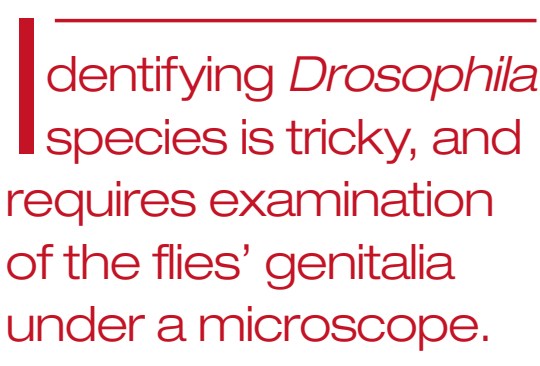

^ ๔ 2000 Macmillan Magazines Ltd 
The report added: "It isn't nice to try to pull the wool over the reviewers' eyes."

Despite such concerns, the review team gave the stock centre a rating of "very good". The scientists involved now acknowledge that many of the centre's users will have greeted this assessment with disbelief. But review-team member Kathleen Matthews, a geneticist who heads the NSF-funded Drosophila Stock Center at the University of Indiana at Bloomington, which maintains about 6,400 different genetic stocks of $D$. melanogaster, says that the reviewers faced a dilemma. If they were too harsh, the facility might have lost its NSF funding. As no one was pushing to take over the centre, there were concerns that the collection would then be lost to science. "We decided to recommend changes to modernize and make the centre function better," says Matthews.

But despite some improvements, problems persisted - until, in 1999, the NSF decided to find the centre a new operator. Even then, Arizona was the only bidder. The NSF will provide Arizona with some \$1 million over the next five years to set up and operate the facility.

But the fact that problems persisted in Bowling Green for so long, and the circumstances of the 1991 review, raise questions about the NSF's funding and appraisal of such centralized facilities. Gerald Selzer, the NSF official responsible for the agency's 17 stock collections of research resources, says that the difficulties have revealed the limitations of the competitive funding process in cases in which academics are not queuing up to run an important facility. The only alternative would be for the NSF to run such facilities as federal labs, for which there is no precedent.

\section{Bright future}

Fruitfly researchers are now looking optimistically to the future. One of Markow's first moves will be to establish a research programme in comparative functional genomics. "The future of the centre is in genomics. It is going to be unbelievable for that type of research," enthuses Spicer, who has collaborated with Markow on comparative studies of Drosophila reproductive $\operatorname{organs}^{5,6}$. Genomic technologies, such as the use of DNA microarrays to chart differences in gene expression, will transform such studies, he says. Markow's thrust into functional genomics has also been boosted by the expectation that the US National Institutes of Health will support the sequencing of the genome of a second Drosophila species: probably D. pseudoobscura (see page 396).

In the spring, Markow intends to host a workshop on Drosophila identification and husbandry, which she hopes will begin to revive the skills needed to curate fruitfly stocks. A new collections manager is also

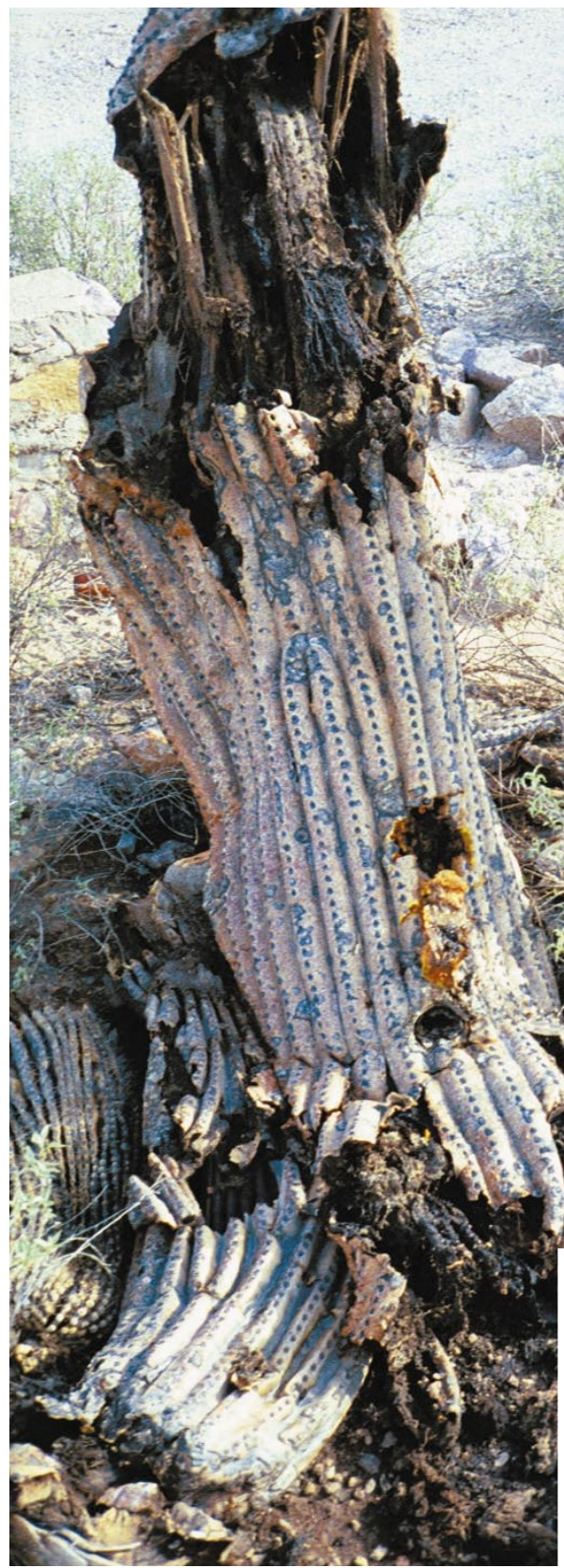

Gotcha: Markow searches the Arizona desert for additions to her stocks of Drosophila.

being trained to operate the stock centre. In the meantime, given the dearth of fly systematists, Markow has enlisted a retired Arizona professor, Bill Heed, to help catalogue the collection. Any contamination of stocks will mean that some species have to be collected again from the wild. But Markow hopes not to stop there, and has plans to add more species to the collection in the near future.

Once the collection has been validated, stocks will be available for ordering over the Internet - another service that was never implemented at Bowling Green. That change is indicative of Markow's new broom, say the facility's users. "The centre is going into amazingly good hands and should be a tremendous resource," says Scott Pitnick,

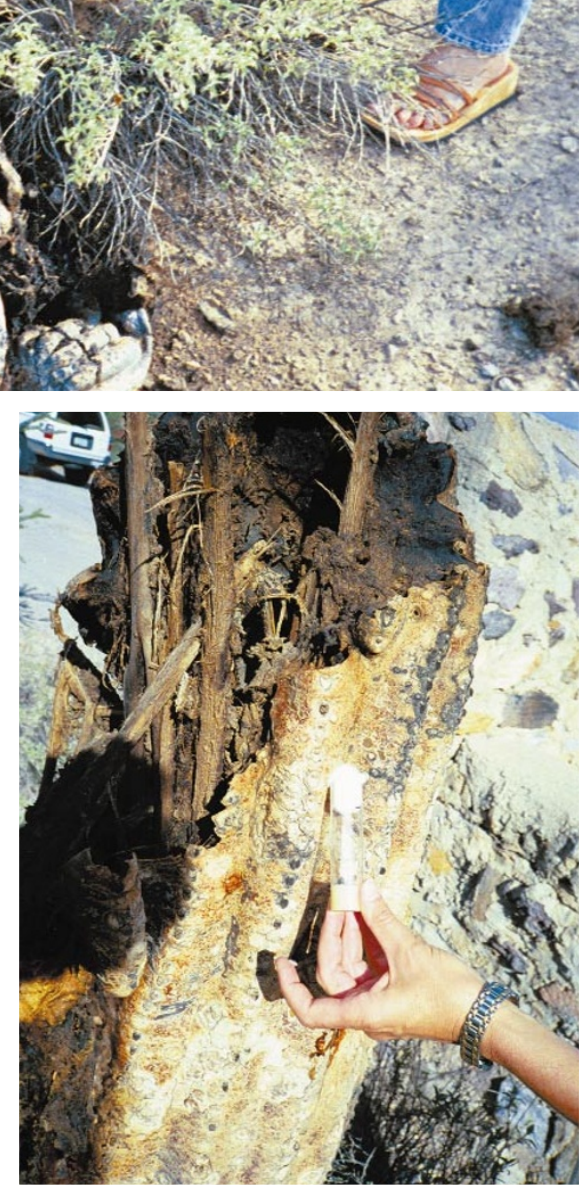

an entomologist at Syracuse University in New York.

Rex Dalton is Nature's West Coast US correspondent.

Adams, M. D. et al. Science 287, 2185-2195 (2000).

2. Bachtrog, D. \& Charlesworth, B. Curr. Biol. 10, 1025-1031 (2000). González, J. et al. Chromosom. Res. 8, 375-385 (2000).

4. Keightley, P. D. \& Eyre-Walker, A. Science 290, 331-333 (2000).

5. Pitnick, S. et al. Evolution 51, 833-845 (1997).

6. Pitnick, S. et al. Evolution 53, 1804-1822 (1999). 